\title{
A NOTE ON THE OPERATORS OF BLASCHKE AND PRIVALOFF
}

\author{
D. H. POTTS
}

Let $f(P)$ be a function of a point $P \equiv P(x, y)$ in Euclidean 2-space. Let $L(f ; P ; r), A(f ; P ; r)$ be the mean values of $f(P)$ on the perimeter and on the interior, respectively, of a circle of center $P$ and radius $r$, that is,

$$
\begin{aligned}
& L(f ; P ; r)=\frac{1}{2 \pi r} \int_{C(P ; r)} f(Q) d s_{Q}, \\
& A(f ; P ; r)=\frac{1}{\pi r^{2}} \iint_{D(P ; r)} f(Q) d Q
\end{aligned}
$$

where $C(P ; r), D(P ; r)$ are the perimeter and interior, respectively, of the circle with center $P$ and radius $r$. The operators

$$
\begin{aligned}
& \nabla_{p} f(P)=\lim _{r \rightarrow 0} \frac{4}{r^{2}}[L(f ; P ; r)-f(P)], \\
& \nabla_{a} f(P)=\lim _{r \rightarrow 0} \frac{8}{r^{2}}[A(f ; P ; r)-f(P)]
\end{aligned}
$$

have been defined by Blaschke and Privaloff, respectively. The following are a few of the results which have been obtained by these and other investigators.

Theorem A $[1,2] .{ }^{1}$ If $f(P)$ has continuous second partial derivatives, then $\nabla_{p} f(P), \nabla_{a} f(P)$ exist, and

$$
\left(\frac{\partial^{2} f}{\partial x^{2}}+\frac{\partial^{2} f}{\partial y^{2}}\right)_{P} \equiv \nabla^{2} f(P)=\nabla_{p} f(P)=\nabla_{a} f(P) .
$$

TheOREM B [1]. If (i) $f(P)$ is continuous on a circle $\bar{D}(Q ; r)$, (ii) $\nabla_{p} f(P)$ exists on the interior, $D(Q ; r)$, then

$$
\frac{4}{r^{2}}[L(f ; Q ; r)-f(Q)]
$$

lies between the upper and lower bounds of $\nabla_{p} f(P)$ on $D(Q ; r)$.

Theorem C $[3,4]$. If $u(P)$ is a logarithmic potential function

Received by the editors July 28, 1947, and, in revised form, September 11, 1947.

1 Numbers in brackets refer to the bibliography at the end of the paper. 


$$
u(P)=\int_{W} \log \frac{1}{P Q} d \mu(Q)
$$

where $\mu$ is a mass distribution, and if the density exists at $R$, that is,

$$
\lim _{r \rightarrow 0} \frac{1}{\pi r^{2}} \int_{D(R ; r)} d \mu(Q)=D_{s} \mu(R)
$$

exists, then $\nabla_{p} u(R), \nabla_{a} u(R)$ exist and $\nabla_{p} u(R)=\nabla_{a} u(R)=-2 \pi D_{s} \mu(R)$. ( $W$ indicates integration over the whole space.)

The purpose of this note is to give extensions of Theorems $\mathrm{B}$ and $\mathrm{C}$. Theorem $\mathrm{B}$ is readily extended to the operator $\nabla_{a}$ by the following:

THEOREM 1. If (i) $f(P)$ is continuous on a circle $\bar{D}(Q ; r)$, (ii) $\nabla_{a} f(P)$ exists on the interior, $D(Q ; r)$, then

$$
\frac{8}{r^{2}}[A(f ; Q ; r)-f(Q)]
$$

lies between the upper and lower bounds of $\nabla_{a} f(P)$ on $D(Q ; r)$.

Proof. Consider the function

$$
\lambda(P)=f(P)-h(P)+L(f ; Q ; \rho)-f(Q)-\frac{1}{\rho^{2}}[L(f ; Q ; \rho)-f(Q)] \overline{P Q}^{2}
$$

where $\rho \leqq r$, and $h(P)$ is the function harmonic on $D(Q ; \rho)$ and such that $h(P)=f(P)$ on $C(Q ; \rho)$. Clearly $\lambda(P)=0$ on $C(Q ; \rho)$. Further $\lambda(Q)=L(f ; Q ; \rho)-h(Q)$. But

$$
h(Q)=\frac{1}{2 \pi r} \int_{C(Q ; \rho)} h(P) d s_{P}=\frac{1}{2 \pi r} \int_{C(Q ; \rho)} f(P) d s_{P}=L(f ; Q ; \rho) .
$$

Therefore $\lambda(Q)=0$. Thus the continuous function $\lambda(P)$ has both a maximum and a minimum value on $D(Q ; \rho)$. Now if $R$ is a maximum point of $\lambda(P)$ then $\nabla_{a} \lambda(R) \leqq 0$, for

$$
\nabla_{a} \lambda(R)=\lim _{\rho \rightarrow 0} \frac{1}{\pi \rho^{4}} \iint_{D(P ; \rho)}[\lambda(P)-\lambda(R)] d P \leqq 0 .
$$

But

$$
\nabla_{a} \lambda(P)=\nabla_{a} f(P)-\nabla_{a} h(P)-\frac{1}{\rho^{2}}[L(f ; Q ; \rho)-f(Q)] \nabla_{a} \bar{P} \bar{Q}^{2} .
$$

By Theorem A, $\nabla_{a} h(P)=0, \nabla_{a} \bar{P}^{2}=4$. Therefore 


$$
\nabla_{a} \lambda(P)=\nabla_{a} f(P)-\frac{4}{\rho^{2}}[L(f ; Q ; \rho)-f(Q)] .
$$

But $\nabla_{a} \lambda(R) \leqq 0$, hence

$$
\nabla_{a} f(R) \leqq \frac{4}{\rho^{2}}[L(f ; Q ; \rho)-f(Q)] .
$$

Similarly if $S$ is a minimum point of $\lambda(P)$ on $D(Q ; \rho)$ we have

$$
\nabla_{a} f(S) \geqq \frac{4}{\rho^{2}}[L(f ; Q ; \rho)-f(Q)] .
$$

Thus, if $M, m$ are the upper and lower bounds, respectively, of $\nabla_{a} f(P)$ on $D(Q ; r)$, then for all $\rho \leqq r$

$$
m \leqq \frac{4}{\rho^{2}}[L(f ; Q ; \rho)-f(Q)] \leqq M,
$$

and so

$$
\frac{2}{r^{2}} \int_{0}^{r} m \rho^{3} d \rho \leqq \frac{8}{r^{2}} \int_{0}^{r} L(f ; Q ; \rho) \rho d \rho-\frac{8}{r^{2}} \int_{0}^{r} f(Q) \rho d \rho \leqq \frac{2}{r^{2}} \int_{0}^{r} M \rho^{3} d \rho .
$$

But

$$
\begin{aligned}
A(f ; Q ; r) & =\frac{1}{\pi r^{2}} \iint_{D(Q ; r)} f(P) d P=\frac{2}{r^{2}} \int_{0}^{r} \rho d \rho \cdot \frac{1}{2 \pi \rho} \int_{C(Q ; \rho)} f(P) d s_{P} \\
& =\frac{2}{r^{2}} \int_{0}^{r} L(f ; Q ; \rho) \rho d \rho .
\end{aligned}
$$

Thus $m r^{2} / 2 \leqq 4[A(f ; Q ; r)-f(Q)] \leqq M r^{2} / 2$ and

$$
m \leqq \frac{8}{r^{2}}[A(f ; Q ; r)-f(Q)] \leqq M .
$$

For the operator $\nabla_{a}$ a somewhat stronger form of Theorem C is obtainable.

THEOREM 2. If $u(P)$ is a logarithmic potential function

$$
u(P)=\int_{W} \log \frac{1}{P Q} d \mu(Q)
$$

where $\mu$ is a mass distribution, and if at $R$ 


$$
\lim _{r \rightarrow 0} \operatorname{ap} \frac{1}{\pi r^{2}} \int_{D(R ; r)} d \mu(P)=D_{a} \mu(R)
$$

exists, then $\nabla_{a} u(R)$ exists, and $\nabla_{a} u(R)=-2 \pi D_{a} \mu(R)$.

Proof. Consider

$$
\begin{aligned}
L(u ; R ; \rho) & =\frac{1}{2 \pi \rho} \int_{C(R ; \rho)} u(P) d s_{P}=\frac{1}{2 \pi \rho} \int_{C(R ; \rho)} d s_{P} \cdot \int_{W} \log \frac{1}{P Q} d \mu(Q) \\
& =\int_{W} d \mu(Q) \cdot \frac{1}{2 \pi \rho} \int_{C(R ; \rho)} \log \frac{1}{P Q} d s_{P} .
\end{aligned}
$$

Now

$$
\begin{aligned}
\frac{1}{2 \pi \rho} \int_{C(R ; \rho)} \log \frac{1}{P Q} d s_{P} & =\log \frac{1}{Q R} & & (Q R>\rho) \\
& =\log \frac{1}{\rho} & & (Q R \leqq \rho) .
\end{aligned}
$$

Hence

$$
\begin{aligned}
L(u ; R ; \rho) & =\int_{D(R ; \rho)} d \mu(Q) \cdot \log \frac{1}{\rho}+\int_{W \rightarrow D} d \mu(Q) \cdot \log \frac{1}{Q R} \\
& =\int_{W} d \mu(Q) \cdot \log \frac{1}{Q R}+\int_{D(R ; \rho)}\left[\log \frac{1}{\rho}-\log \frac{1}{Q R}\right] d \mu(Q) \\
& =u(R)+\int_{D(R ; \rho)}\left[\log \frac{1}{\rho}-\log \frac{1}{Q R}\right] d \mu(Q) .
\end{aligned}
$$

Thus

$$
\begin{aligned}
A(u ; R ; r) & =\frac{2}{r^{2}} \int_{0}^{r} L(u ; R ; \rho) \rho d \rho \\
& =u(R)+\frac{2}{r^{2}} \int_{0}^{r} \rho d \rho \cdot \int_{D(R ; \rho)}\left[\log \frac{1}{\rho}-\log \frac{1}{Q R}\right] d \mu(Q),
\end{aligned}
$$

and so

$$
\begin{aligned}
& \frac{8}{r^{2}}[A(u ; R ; r)-u(R)] \\
& =\frac{16}{r^{4}} \int_{0}^{r} \rho d \rho \cdot \int_{D(R ; \rho)}\left[\log \frac{1}{\rho}-\log \frac{1}{Q R}\right] d \mu(Q) .
\end{aligned}
$$


The integrand depends only on $|Q R|$, so we can write

$$
\frac{8}{r^{2}}[A(u ; R ; r)-u(R)]=\frac{16}{r^{4}} \int_{0}^{r} \rho d \rho \cdot \int_{0}^{\rho}\left[\log \frac{1}{\rho}-\log \frac{1}{t}\right] d \bar{\mu}(t)
$$

where

$$
\bar{\mu}(t)=\int_{D(R ; t)} d \mu(Q)
$$

Integrating by parts we have

$$
\begin{aligned}
\frac{8}{r^{2}}[A(u ; R ; r) & -u(R)] \\
= & \frac{16}{r^{4}} \int_{0}^{r} \rho d \rho \cdot\left\{\left[\left(\log \frac{1}{\rho}-\log \frac{1}{t}\right) \bar{\mu}(t)\right]_{0}^{\rho}-\int_{0}^{\rho} \bar{\mu}(t) \frac{d t}{t}\right\} .
\end{aligned}
$$

But

$$
\bar{\mu}(t)=\int_{D(R ; t)} d \mu(Q)=\pi t^{2} D_{a} \mu(R)+o\left(t^{2}\right)
$$

for almost all small $t$. Hence

$$
\begin{aligned}
\frac{8}{r^{2}}[A(u ; R ; r) & -u(R)] \\
& =\frac{16}{r^{4}} \int_{0}^{r} \rho d \rho \cdot\left\{-\int_{0}^{\rho} \pi t^{2} D_{a} \mu(R) \frac{d t}{t}-\int_{0}^{\rho} o\left(t^{2}\right) \frac{d t}{t}\right\} \\
& =\frac{16}{r^{4}} \int_{0}^{r} \rho d \rho \cdot\left[-\frac{\pi}{2} \rho^{2} D_{a} \mu(R)+o\left(\rho^{2}\right)\right] \\
& =-2 \pi D_{a} \mu(R)+\frac{16}{r^{4}} \int_{0}^{r} o\left(\rho^{3}\right) d \rho .
\end{aligned}
$$

Thus

$$
\lim _{r \rightarrow 0} \frac{8}{r^{2}}[A(u ; R ; r)-u(R)]=\nabla_{a} u(R)=-2 \pi D_{a} \mu(R) .
$$

Many results which have been proven for one operator can be extended to the other operator by use of the following theorem.

Theorem 3. If $\nabla_{p} f(P)$ exists, then so does $\nabla_{a} f(P)$, and $\nabla_{a} f(P)$ $=\nabla_{p} f(P)$. 
Proof. $L(f ; P ; r)$ exists for small $r$, and further

$$
L(f ; P ; r)=f(P)+\frac{r^{2}}{4} \nabla_{p} f(P)+o\left(r^{2}\right) .
$$

Also

$$
A(f ; P ; r)=\frac{2}{r^{2}} \int_{0}^{r} L(f ; P ; \rho) \rho d \rho .
$$

And hence

$$
\begin{aligned}
\frac{8}{r^{2}}[A(f ; P ; r)-f(P)] \\
=\frac{8}{r^{4}}\left[2 \int_{0}^{r} L(f ; P ; \rho) \rho d \rho-r^{2} f(P)\right] \\
=\frac{8}{r^{4}}\left\{2 \int_{0}^{r}\left[f(P)+\frac{\rho^{2}}{4} \nabla_{p} f(P)+o\left(\rho^{2}\right)\right] \rho d \rho-r^{2} f(P)\right\} \\
=\nabla_{p} f(P)+\frac{16}{r^{4}} \int_{0}^{r} o\left(\rho^{3}\right) d \rho .
\end{aligned}
$$

The last term is easily seen to approach zero as $r \rightarrow 0$. Thus

$$
\lim _{r \rightarrow 0} \frac{8}{r^{2}}[A(f ; P ; r)-f(P)]=\nabla_{a} f(P)=\nabla_{p} f(P) .
$$

These results hold true for spaces of higher dimensions, and the above proofs follow through with obvious modifications of the coefficients and the form of the potential function.

\section{BIBLIOGRAPHY}

1. W. Blaschke, Ein Mittelwertsatz und eine kennzeichende Eigenschaft des logarithmischen Potentials, Berichte über die Verhandlungen der königlich sachsischen Gesellschaft der Wissenschaften zu Leipzig, Mathematisch-Physische Klasse vol. 68 (1916) pp. 3-7..

2. I. Privaloff, Sur les functions harmonique, Rec. Math. (Mat. Sbornik) vol. 32 (1924-1925) pp. 464-469.

3. - On a theorem of S. Saks, Rec. Math. (Mat. Sbornik) N.S. vol. 9 (1941) pp. 457-460.

4. S. Saks, On the operators of Blaschke and Privaloff for subharmonic functions, Rec. Math. (Mat. Sbornik) N.S. vol. 9 (1941) pp. 451-456.

NORTHWESTERN UNIVERSITY 